\title{
Assembly and metrology of NIF target subassemblies using robotic systems
}

\author{
K.-J. Boehm, N. Alexander, J. Anderson, L. Carlson, and M. Farrell \\ General Atomics, P.O. Box 85608, San Diego, CA 92186-5608, USA \\ (Received 30 March 2017; revised 27 May 2017; accepted 20 July 2017)
}

\begin{abstract}
With European Laser Facilities such as the Extreme Light Infrastructure (ELI) and the Helmholtz International Beamline for Extreme Fields (HIBEF) scheduled to come online within the next couple of years, General Atomics, as a major supplier of targets and target components for the High Energy Density Physics community in the United States, is gearing up to meet their demand for large numbers of low cost targets. Using the production of a subassembly for the National Ignition Facility's fusion targets as an example, we demonstrate that through automation of assembly tasks, the design of targets and their experimental setup can be fairly complex while keeping the assembly time and cost as a minimum. A six-axis Mitsubishi robot is used in combination with vision feedback and a force-torque sensor to assemble target subassemblies of different scales and designs with minimal change of tooling, allowing for design flexibility and short assembly setup times. Implementing automated measurement routines on a Nikon NEXIV microscope further reduces the effort required for target metrology, while electronic data collection and transfer complete a streamlined target production operation that can be adapted to a large variety of target designs.
\end{abstract}

Keywords: assembly; automation; hohlraum; robotics; target

\section{Introduction}

Shot rates of $0.1-10 \mathrm{~Hz}$ on some of the new laser facilities in Europe [e.g., the Extreme Light Infrastructure (ELI) and the Helmholtz International Beamline for Extreme Fields (HIBEF)] will require target production in quantities from dozens to thousands per campaign. These targets need to be precision assembled and subsequently measured. Currently, both operations are done under a microscope by hand. This labor cost is the main contributor to the total cost of a target $^{[1]}$.

Motivated by the ramp-up of cryogenic fusion experiments on the National Ignition Facility (NIF), General Atomics (GA) started automating the component assembly of targets, see Ref. [2]. Target components with a stable design, such as the thermomechanical packaging (TMP) and the silicon cooling arms (shown in Figure 1), lend themselves to robotic assembly as described in Ref. [2]. Assembly of components that vary in design are more challenging to automate; however, the versatility in target design is important to supply the Physics community with enough flexibility in their quest to achieve fusion ignition at the NIF.

The process of inserting gold hohlraums (HRs) with varying sizes and designs into the TMP is presented in this

Correspondence to: K.-J. Boehm. Email: boehm@fusion.gat.com

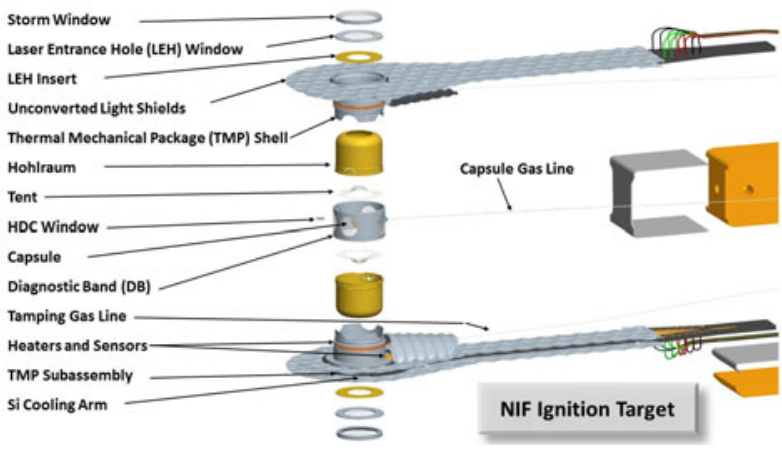

Figure 1. A typical NIF target is shown in an exploded view. The work presented here focuses on the assembly of the HR into the TMP subassembly. Each NIF target requires two of these, one upper and one lower assembly.

paper as an example in which a process involving a flexible component design can be automated. Currently, a highly trained technician can insert a gold HR in roughly $2.5 \mathrm{~h}$ including measuring and reporting the data. The automated process discussed in this paper does the same in about $30 \mathrm{~min}$.

Section 2 describes the details of the HR insertion process; Section 3 describes the automated metrology and outlines the results achieved to date using this setup. Section 4 illustrates 


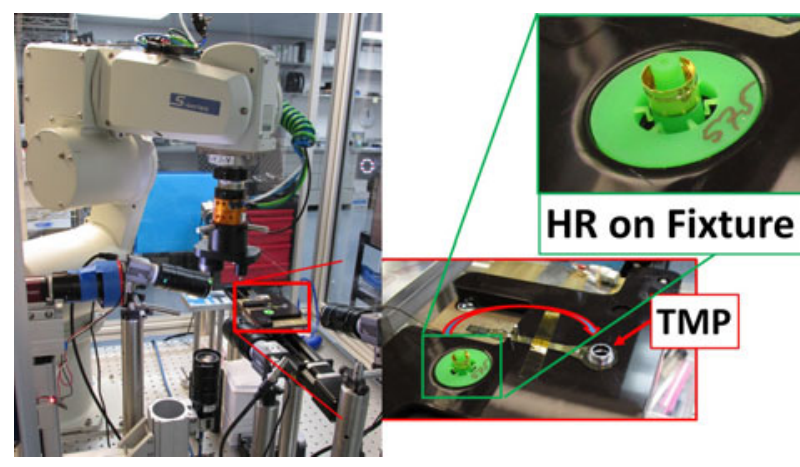

Figure 2. The robot cell used for the work presented in this paper is depicted on the left. On the right, in the zoomed-in images, the components to be assembled are shown.

the impact of automation on the field of target fabrication especially in the context of target design for large quantities as well as restrictions and requirements based on the lessons learned from the current system.

\section{Setup and process for automated HR insertion}

In general, robotic automation is implemented in processes in which relatively large quantities of the same design are to be produced or assembled justifying the development of tooling and robot programs by significant cost or time savings. Due to the relatively low number of identical targets produced for experiments on the NIF (rarely more than 4 units are of the exact same type), automation of target production processes requires individual customization.

The robot system developed for the HR insertion process described in this work is depicted in Figure 2. It features a Mitsubishi six-axis robotic arm (shown on the left of Figure 2) equipped with a force-torque (F-T) sensor at the 'wrist'. A 3D printed dual end effector is mounted to the F-T sensor, featuring a rubber stamp to apply glue and a vacuum chuck for the pick and place operations. The robot movements are programmed in the native language of the robot controller, but are commanded through a PC-based LabVIEW user interface.

A linear stage with $300 \mathrm{~mm}$ of travel is mounted on the optical table to present the specimens to the robot. Multiple HR-TMP pairs can be lined up on that stage and can be driven to the center of the work cell for sequential insertion. Only one pair has been used to date, an example of this is shown on the right in Figure 2.

Also on the optical table is a vision system using two orthogonal cameras used to position the HR to the correct clocking angle as described below and depicted in Figure 3. National Instrument's Vision software is used to acquire and process the images as well as to perform the required calculations.

The two photos on the right in Figure 2 show how the HR and TMP pair is placed on 3D printed fixtures and presented

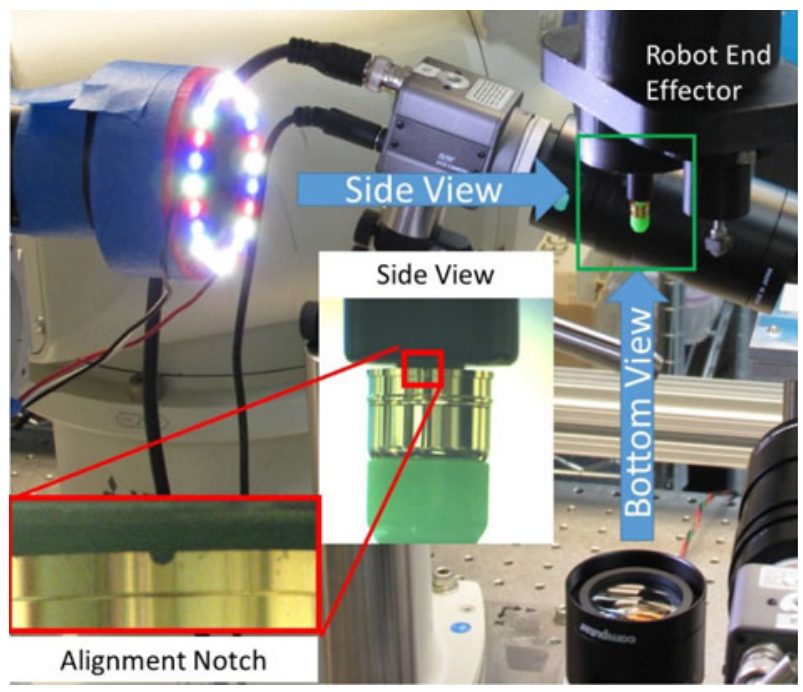

Figure 3. The fill tube notch on the upper edge of the HR serves as an alignment fiducial to rotate the HR to the correct clocking angle. Two orthogonal cameras are used to correct for fixture tolerances, robot arm position inaccuracies and HR placement errors.

to the robot. This system is used to glue the HR into the TMP with a position accuracy of a few microns (in all three lateral dimensions) and a rotational accuracy of $0.5^{\circ}$ around the cylinders center axis. A force of about $100 \mathrm{~g}$ is applied onto the HR while the glue is being cured in order to keep the glue gap below $5 \mu \mathrm{m}$. More details on how these accuracies are achieved on these parts using this system are presented in Section 3.

In order to benefit from automating this process, the robot system needs to be able to insert a large variety of HR designs without having to change the robot program or the physical setup of the robot work cell. This flexibility is achieved by:

(1) generalizing the robot program - all HR designs can be inserted as long as they meet basic NIF cryogenic target design specifications;

(2) using 3D printed fixtures to accommodate design changes of target components as these can be produced quickly and inexpensively (the fixtures must interface with the rest of the setup and be easily exchangeable);

(3) implementing a feedback loop with an F-T sensor such that surfaces can be approached even if their exact locations are not specified (see Figure 4);

(4) relying on design features common throughout the cryogenic target platforms such as the location and dimension of the fill tube notch (see Figure 3).

The specifics of the insertion process are described below. After manually loading the HR and the TMP onto a semikinematic fixture, it is placed into the working cell of the 


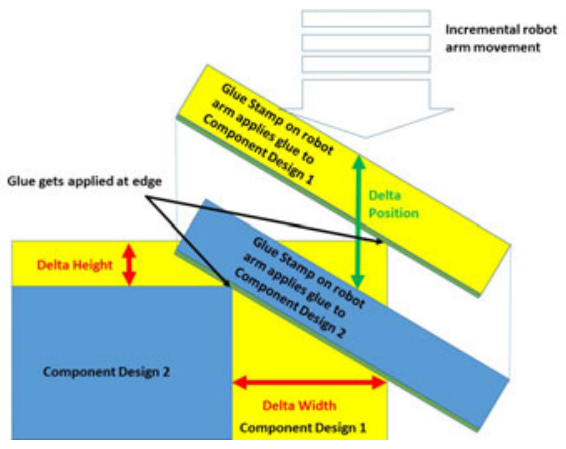

Figure 4. The robot arm approaches a surface in small increments (downwards) until contact is detected by the F-T sensor. This sketch shows how the same stamping tool can be used to apply glue on two different component designs without changing the robot program.

robot system. Multiple TMPs and HRs could be loaded on a tray side by side as has been done in Ref. [2]. The linear stage could then be used to move each pair into the center of the robot cell for assembly; however, in this proof of concept, only one $\mathrm{HR}$ is inserted at a time. A two-piece set of 3D printed tools (shown in Figure 2 on the far right) is used to pick up the HR of a specific design. This tool can easily be adapted to different HR designs and can be produced quickly and inexpensively. This tool set is inserted into the semikinematic fixture prior to loading the HR.

Using the rubber stamp on the robot end effector a small amount of UV-glue (less than $5 \mathrm{~nL}$ ) is applied onto the aluminum rim of the TMP that the HR will sit on. Since the exact location of the mating surface varies with the HR's diameter, the stamp is angled and gets driven down until the F-T sensor is activated as shown in Figure 4. Using the vacuum tip on the end effector, the robot arm picks up a 3D printed tool which supports the HR and allows transfer of the HR without touching any of the HRs surfaces.

Next, the HR must be rotated into the proper orientation before insertion into the TMP. A small feature on the HR, the notch which holds the capsule fill tube (see Figure 1), is being used as a fiducial for orientation detection. As the size and shape of that fill tube notch (a semi-circular trough with a $0.1 \mathrm{~mm}$ radius, see Figure 3 ) remains constant throughout all HR designs, a pattern searching algorithm in LabVIEW can be used to locate it.

The tight tolerances on the clocking angle of the finished assembly $\left( \pm 0.5^{\circ}\right)$ to the TMP axis requires the use of a dual camera system. The tolerance stack-up between the accuracy of the 3D printed fixtures, the repeatability of the robot arm position and the positioning of the HR on the fixture are sufficient to introduce an uncertainty larger than the assembly spec if only one camera was being used. In the dual camera system, one camera looks at the HR end-on from below to find the center of the cylinder. The second camera locates the location of the fill tube notch in the horizontal

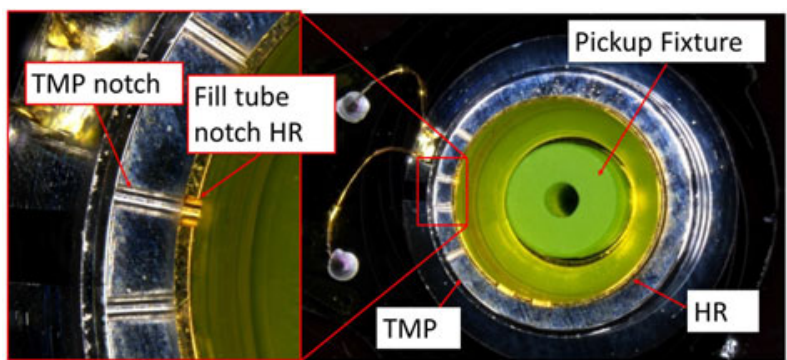

Figure 5. Top view of an inserted HR with the 3D printed fixture still in the center of the assembly. The TMP notches serve as an alignment verification fiducial.

plane so that a rotation correction can be calculated. In a calibration step performed during setup, the vision system is tuned to determine the correction parameters.

As a next step, the robot moves the HR just above the TMP. After small adjustments of the alignment of the HR to the TMP in the lateral directions, the HR is slowly lowered into the TMP. The vacuum is released, and the HR tool falls through the TMP into a small void in the fixture underneath the TMP opening.

Using the F-T sensor, the robot arm can gently approach the end effector to the upper edge of the HR and then apply a force of $1-1.5 \mathrm{~N}$ to ensure proper seating of the HR into the TMP. During this step, the glue between the two parts is squeezed out leaving the parts bonded with a minimal glue bond thickness, see Figure 5.

The glue is cured using a hand-held UV pen while the robot arm is applying force holding the two parts together.

\section{Metrology and results}

The two most important metrics for a successful insertion are the insertion depth and the clocking of the HR with respect to the TMP. While the former is important to ensure that the two HR halves do not interfere with one another during closing of the target (see Figure 1), the latter is important to ensure that diagnostic window cutouts on the two HR halves align with one another.

Both the alignment and the insertion depth can be measured using a NEXIV optical coordinate measurement microscope (OCMM) like the one shown in Figure 6. The specimen is placed on an $X-Y$ microscope slide with a 300 $\mathrm{mm} \times 200 \mathrm{~mm}$ travel. The microscope's optical system is able to image specimens using a CCD video camera with sub-micron pixel resolution, while a laser probe delivers submicron height measurements. Measurements are taken by using Nikon's VMZ-R Automeasure software based on the images from the CCD camera. The software enables repeated measurements to be performed following automated measurement routines called recipes. 


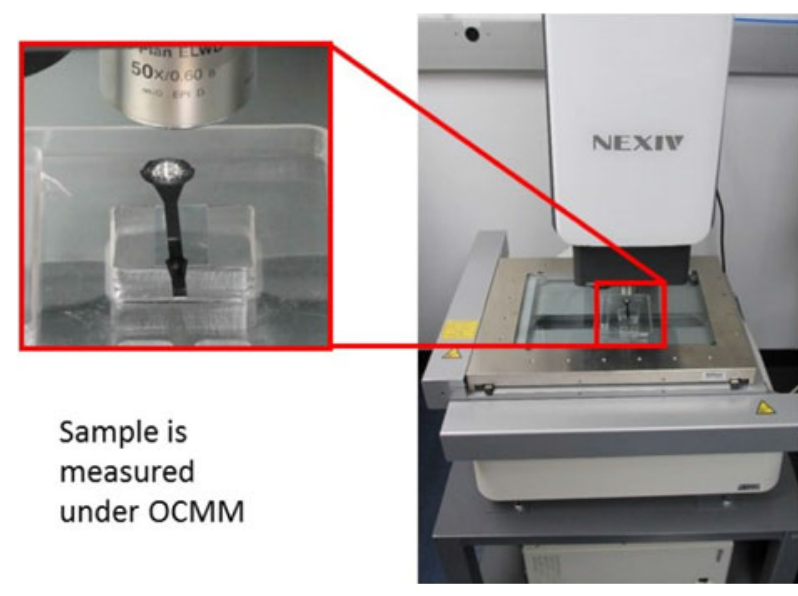

Figure 6. A photo of the OCMM microscope is shown. The sample is placed on the microscope slide and measured using pre-programmed recipes.

Using such an automated routine on a NEXIV VMZ machine, both the clocking of the HR inside the TMP (see Figure 7) and the insertion depth (see Figure 8) are measured. The glue bond thickness can be calculated from the insertion depth and the metrology data of the individual components. The entire measurement routine takes about 2 min to set up and $2 \mathrm{~min}$ to run. The OCMM is programmed to measure the assembly using the following strategy.

Two locations on the silicone arm are used to define the coordinate system of the metrology routine (see Figure 7). This seems like a good choice since the features on the silicone arms are produced with sub-micron accuracy and the wafers are remarkably flat. The midpoint between the two spots marks the location of the origin, the vector between the two is set as the orientation of the $x$-axis. In order to fully define the orientation of the coordinate system, the microscope will take a number of measurements of the datum plane on the can (see Figure 8) and set the orientation of the $z$-axis perpendicular to that plane, thus correcting for angular misalignment between the specimen and the microscope stage.

The height is determined by measuring the height along the top surface of the HR and measuring the distance to the datum plane.

The clocking angles between the three components (silicone arm, can and HR) are measured by fitting straight lines along features on the parts that provide a strong black/white contrast on the microscope image as illustrated in Figure 7.

The repeatability of both the robotic insertion as well as the measurement system needed to be determined. Since both the HR and the TMP both have highly reflective surface finishes, even minute changes in light condition between measurements are expected to change the measurement result. Rather than relying on the manufacturer's specifications, the repeatability of the measurement microscope was determined first. It is important to notice that the results quoted in this work are measured for the specific target design used for these tests. Especially when it comes to clocking accuracy, different HR sizes might yield different repeatability results, both for robotic insertion and subsequent measurements.

A total number of ten measurements were taken using the same HR-TMP assembly. Five of these measurement were taken without moving the sample and five were taken at different locations on the OCMM slide. Based on these results, it was determined that the repeatability of the microscope measuring the insertion depth of this sample was below the $0.3 \mu \mathrm{m}$ specified by the OCMM manufacturer independent of whether the sample was moved or not. For the clocking angle, it was found that the repeatability of the measurement was $0.01^{\circ}$ if the sample is stationary (in the same location on the microscope stage) and $0.06^{\circ}$ if the sample is moved around the stage. Small changes in the lighting conditions and the surface texture of the parts are most likely responsible for this result.

Next, the repeatability of the robot insertion was measured. For this test, the HR was released from the TMP and re-inserted using the procedure outlined in Section 2. After reassembly, the part was measured at two different locations on the microscope stage. This process was repeated five times. The test results for the clocking measurements are shown in Figure 9. The solid line represents the HR clocking measurement for the same assembly taken at the same location for test numbers one through five and at different locations on the microscope slide for tests six through ten. The dots represent the pair of measurements taken after the HR was removed from the TMP and re-inserted using the robot arm. The robotic insertion imposes a bias on the data, currently $0.23^{\circ}$, which is attributed to a misalignment of the 2-axis camera system to the TMP fixture on the optical table (setup shown in Figure 2). As more data is collected on this system, the bias can be corrected for. One standard deviation of the dataset is considered the insertion uncertainty.

The insertion depth measured for these assemblies is less a measure of the robot's placement accuracy as it is a measure of the glue bond thickness. The HR's are driven by the robot arm against a hard stop made up by a step designed into the two parts to be mated. The F-T sensor ensures that the components are held together while the glue is being cured using a UV light source. Similarly, a torus machined into the outside wall of the HR is designed to guide the HR into position to a very tight fit ( $3 \mu \mathrm{m}$ radial clearance between the HR and the TMP shell). The fixtures used to hold the two components during assembly must allow for a small shift between the two components as they are mated, since the robot arm has a manufacturer's specification of only $\pm 20 \mu \mathrm{m}$ in placement accuracy. The results from the glue thickness measurements are presented in Table 1; the 

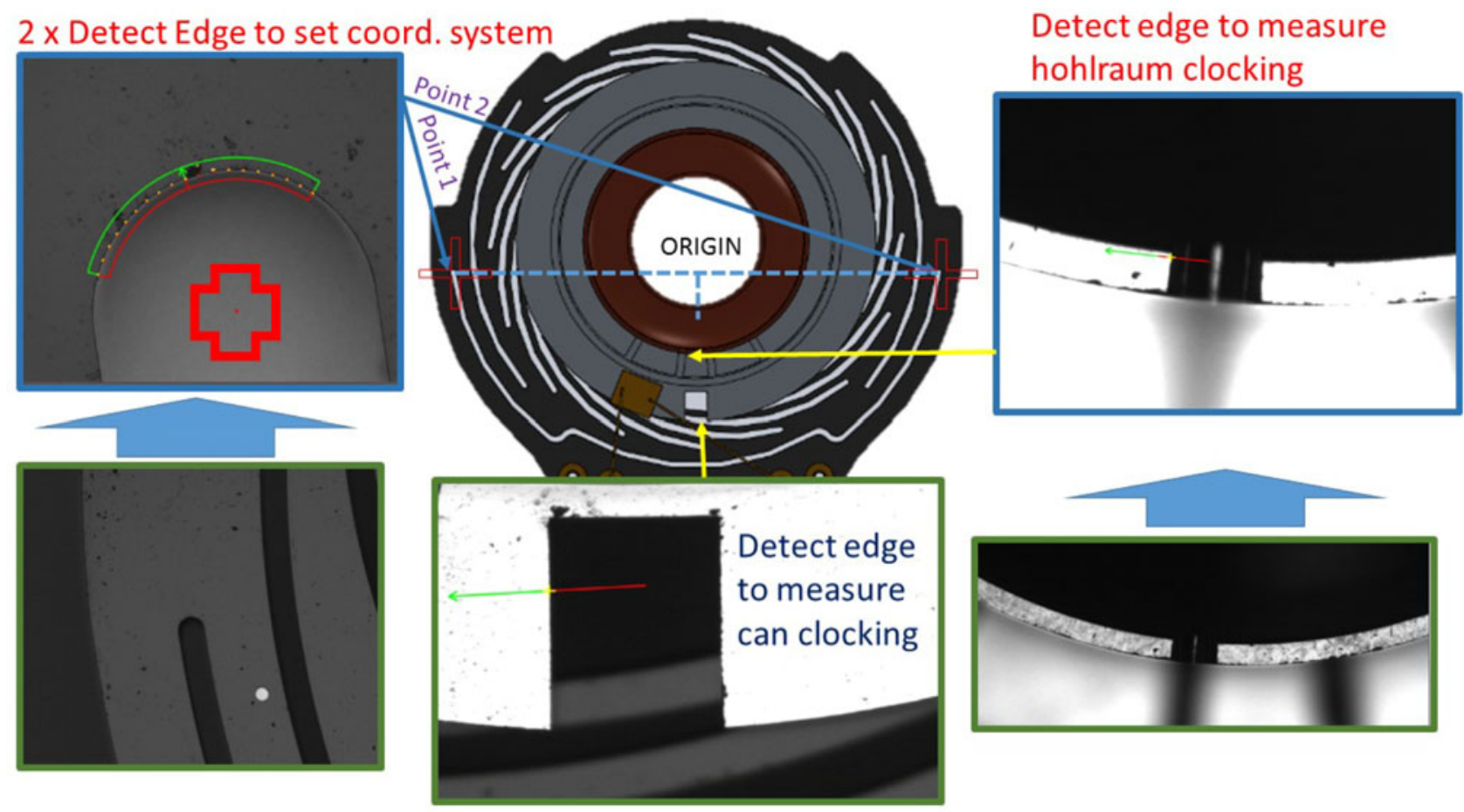

Figure 7. Screen shots from the OCMM metrology routine show measurements of clocking angles on the sample.

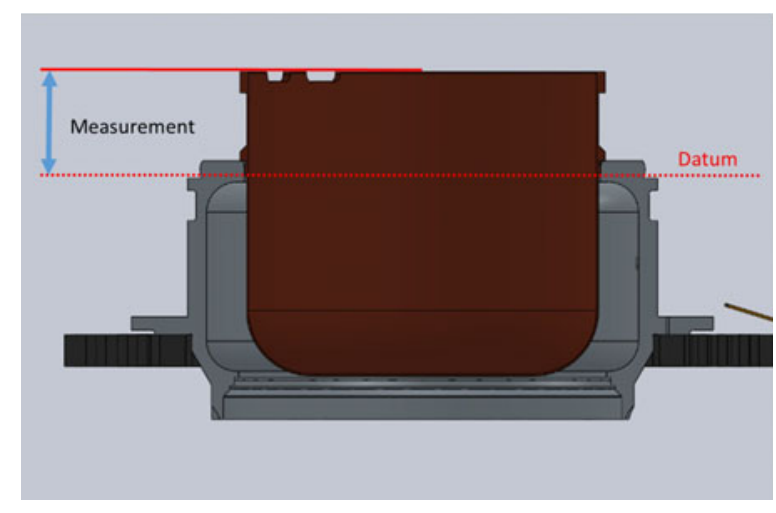

Figure 8. The HR insertion depth is measured by determining the height of the HR rim over the TMP can datum as shown in this cross-section.

Table 1. Accuracy for the HR insertion and metrology processes.

\begin{tabular}{lcc}
\hline Standard deviation & $\begin{array}{c}\text { Clocking angle } \\
\text { (degrees) }\end{array}$ & $\begin{array}{c}\text { Insertion depth } \\
(\mu \mathrm{m})\end{array}$ \\
\hline Metrology (same loc.) & 0.01 & 0.3 \\
Metrology (diff. loc.) & 0.06 & 0.3 \\
Robotic insertion & $0.43 \pm 0.06$ & $1.5 \pm 0.3$ \\
\hline
\end{tabular}

radial alignment between the HR and the TMP is considered sufficient if the two parts fit together due to their tight machining tolerances.

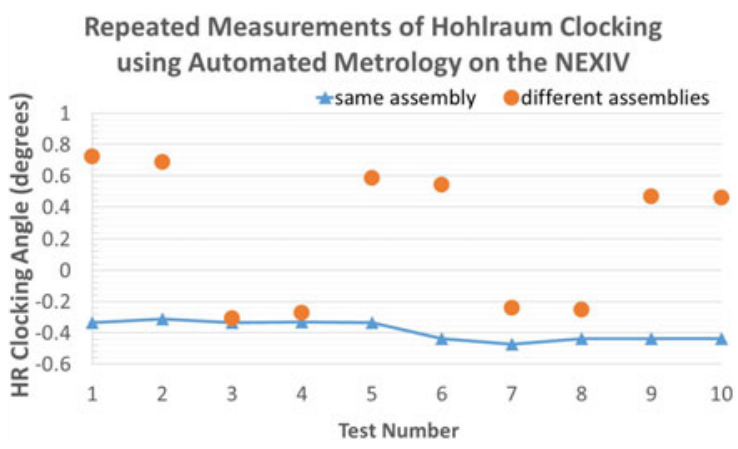

Figure 9. The raw data from the HR clocking measurements is presented. Repeated measurements of the clocking angle of the same part gives the expected error of the measurement, while data taken on different assemblies give a measure of repeatability of the robotic insertion process.

\section{Outlook and perspective}

The introduction of automation or motorized assistance of target fabrication tasks into high energy density (HED) target fabrication tasks is by far not novel, see Ref. [3] as well as Refs. $[4,5]$. The difference to Lee's work is that the process presented here allows assembly of target components of different designs without changing robot programs or setting up new hardware. The difference to Montesani's 'Robotic Assembly Machine' is that the manipulation of components is done by one robot arm rather than a series of 19 automatic and ten manual stages. Of course, the level of system 
complexity is driven by the nature of the assembly process such as the number of parts to be assembled at the same time.

The robotic HR assembly process reduces the overall cost of NIF target production and provides an example of the target complexity that can be accommodated while still employing automation.

In contrast to the expected demand for targets for the European HED facilities, current demand of NIF targets is approximately one target per day. However, it is advantageous to put processes in place that are easy to scale up as the shot rate is expected to increase over the next couple of years. Furthermore, GA is motivated to better use skilled technicians and relieve them from mundane tasks.

Development of this process took about 6 months, which falls well within the time frame of preparation for an HED experiment. Especially if the production team is involved early in the design process, vision recognizable features as well as robot tooling interfacing with the components can be developed and tested in parallel.

\section{Conclusion}

The presentation of the HR insertion process and subsequent metrology demonstrates the feasibility of robotic assembly and qualification measurements of HED Physics target subassemblies.

The ability to use automation to precision assemble targets as presented in this work could have a deep impact on the design of HED targets for high-repetition rated facilities.

Using a robot system equipped with the senses of touch and vision through F-T and camera sensors along with image recognition software, allows for fairly complex planar as well as 3D target designs while alleviating the need for labor intensive assembly processes. The automation of target production processes such as the one demonstrated in this paper could have a major impact on the physical complexity of targets to be shot at high-rep rated facilities.

Target engineers, physicists and users of HED Physics facilities can consider these technologies when balancing the complexity of their experiments with the anticipated cost of target production.

\section{Acknowledgements}

This work was performed under the auspices of the U.S. Department of Energy by General Atomics under Contract DE-NA0001808 and General Atomics IR\&D Funds.

\section{References}

1. E. T. Alger, J. Kroll, E. G. Dzenitis, R. Montesanti, J. Hughes, M. Swisher, J. Taylor, K. Segraves, D. M. Lord, J. Reynolds, C. Castro, and G. Edwards, Fusion Sci. Technol. 59, 78 (2011).

2. L. Carlson, H. Huang, N. Alexander, J. Bousquet, M. Farrell, and A. Nikroo, Fusion Sci. Technol. 70, 274 (2016).

3. G. E. Lee, N. B. Alexander, E. Diaz, and J. D. Sheliak, Fusion Sci. Technol. 59, 227 (2011).

4. R. C. Montesani, R. M. Seugling, J. L. Klingmann, E. G. Dzenitis, E. T. Alger, G. L. Miller, R. A. Kent, C. Castro, J. L. Reynolds, and M. A. Carrillo, in Proceedings of the American Society of Precision Engineering 2008 Annual Meeting (2008).

5. R. C. Montesani, E. T. Alger, L. J. Atherton, S. D. Bhandarkar, C. Castro, E. G. Dzenitis, G. J. Edwards, A. V. Hamza, J. L. Klingmann, D. M. Lord, A. Nikroo, T. G. Parham, J. L. Reynolds, R. M. Seugling, M. Stadermann, M. F. Swisher, J. S. Taylor, and P. J. Wegner, Fusion Sci. Technol. 59, 70 (2010). 